\title{
Wer kann kommen? Ethnische Einwanderung in Israel und Deutschland?
}

\author{
Dietrich Thränhardt
}

Angenommen: 23. September 2021 / Online publiziert: 13. Oktober 2021

(C) Der/die Autor(en) 2021

Panagiotidis, Jannis (2019): The Unchosen Ones: Diaspora, Nation, and Migration in Israel and Germany. Bloomington: Indiana University Press. 380 Seiten. $\$ 40$

Panagiotidis, Jannis (2021): Postsowjetische Migration in Deutschland. Eine Einführung. Mit einem Vorwort von Sergej Lagodinsky. Weinheim: Beltz Juventa. 246 Seiten. $19,95 €$

Wer ist Jude? Wer ist Deutscher? Diese Fragen stellten sich, als Israel mit dem „Rückkehrgesetz“ und Deutschland mit der Aussiedlerregelung ethnische Zuwanderung organisierten. Jannis Panagiotidis untersucht in „The Unchosen Ones“ anhand der israelischen und deutschen Akten, wer abgelehnt wurde, warum und wie das geschah, wer Einfluss nahm, wie Entscheidungen begründet und revidiert wurden und welches nationale Selbstverständnis dahinterstand. Einzelne Schicksale zeigen exemplarisch, wie einschneidend bürokratische Entscheidungen sein könnten. $\mathrm{Pa}$ nagiotidis verfolgt die Erweiterungen und Einschränkungen bei der Aufnahme über sieben Jahrzehnte bis hin zum paradoxen Ende der Entwicklung, als die Mehrheit der ,ethnischen“ Zuwanderer nichtjüdische bzw. nichtdeutsche Familienangehörige waren.

Die schrittweise Öffnung der Sowjetunion, die mit „Korb 3“ des Vertrags über Sicherheit und Zusammenarbeit in Europa 1975 begonnen hatte, löste eine Dynamik aus, die drei Millionen Deutsche und eine Million Juden erfasste. In dieser Zeit ergab sich die deutlichste Parallelität zwischen den Aufnahmeprozessen in den beiden Ländern, eingebunden in die Entspannungspolitik zwischen Ost und West. Angesichts der anfangs restriktiven sowjetischen Auswanderungsregelungen orientierten

Dietrich Thränhardt $(\bowtie)$

Institut für Politikwissenschaft, Universität Münster, Münster, Deutschland

E-Mail: thranha@uni-muenster.de 
sich beide Länder an dem innersowjetischen Passsystem, mit dem Sowjetbürger als deutsch bzw. jüdisch gekennzeichnet wurden. Die Diskrepanz zwischen der religiösen jüdischen Definition von Juden als Kind einer jüdischen Mutter und der sowjetischen Praxis der Weitergabe durch den Vater und den Vatersnamen wurde in Israel expansiv gelöst, während Deutschland auf Wunsch des Zentralrats der Juden die religiöse Definition zugrunde legte und damit die jüdische Zuwanderung einschränkte, parallel zur weitgehenden Beendigung des Aussiedlerzuzugs 2005. Damit war die Parallelität beendet. Deutschland begrenzte die Zuwanderung aus der Sowjetunion 1993-2005 schrittweise, in Israel lief sie mit der Erschöpfung des Potenzials weitgehend aus. Gestaltete sich die Auswanderung schwierig (Ostblock bis 1989, arabische Länder im Kriegszustand mit Israel), waren beide Länder großzügig mit der Aufnahme. War die Auswanderung offen, so setzten Selektivitäten ein.

Trotz dieser Parallelität waren die Aufnahmekulturen in Israel und Deutschland sehr unterschiedlich. Panagiotidis stellt den deutschen ,ethno-humanitarian welfare state“ (S. 21) dem ,israelischen Aufbaustaat“ gegenüber. „An ideal Aussiedler was an endogamous, German speaking victim of Communism with no specific age and no predetermined social features. An ideal Oleh, by contrast, was - apart from being Jewish - healthy, young, and able to work, or alternatively at least economically well-to-do“ (S. 21). Während die Bundesrepublik Aussiedlerinnen und Aussiedlern ein breites Paket wohlfahrtsstaatlicher Leistungen bot, bis hin zur Anrechnung der Rentenjahre in der Sozialversicherung, operierte Israel in den ersten Jahrzehnten mit einem System gesundheitlicher und sozialer Selektivitäten. Dies traf besonders marokkanische Jüdinnen und Juden während der Kolonialzeit bis 1956, als israelische Kommissionen die Auswanderung regelten, ohne dass marokkanische Behörden Einfluss nehmen konnten. „The state of Israel is not a charitable institution, it is a building enterprise“, erklärte der israelische Premierminister Moshe Sharett 1954. Man wollte „working and fighting hands“(S. 21), Kranke standen vor einer „medical border“ (S. 85). Bis heute prägen diese Unterschiede die deutsche und die israelische Integrationspolitik. Während Israel seine jüdischen Zuwanderer direkt zu aktiven Bürgerinnen und Bürgern machen will und seine Hilfen dementsprechend strukturiert, gibt Deutschland viel Geld für Sozialleistungen aus, ist aber weniger erfolgreich bei der wirtschaftlichen Integration.

In beiden Ländern gab es spezielle Bürokratien und Interessengruppen, die die Zuwanderung beeinflussten. In Deutschland traten die Vertriebenenverbände für solidarische Offenhaltung der Aufnahme ein gegenüber einer zunehmend gleichgültiger werdenden Öffentlichkeit. In der Anfangszeit gab es aber auch Einsprüche gegen Bewerberinnen und Bewerber, deren Deutschsein bestritten wurde. Dazu gehörten auch Juden aus dem Ostblock, die zwar deutsch sprachen, aber nach den statistischen Kriterien des Herkunftslandes nicht als Deutsche, sondern als Juden klassifiziert waren. Deutschland schuf 1989/90 dann einen besonderen Einwanderungskanal für Jüdinnen und Juden aus der Sowjetunion. Es entwickelten sich Narrative, die ,jüdischen Kontingentflüchtlingen" und Aussiedlern unterschiedliche Eigenschaften zuschrieben: Intellektualität auf der einen Seite, konservative Zuverlässigkeit auf der anderen. In Israel war das säkulare Establishment maßgebend für die Zuwanderung aus Osteuropa, wo auch die politischen Führungsschichten ursprünglich herstammten. Für die späte Zuwanderung aus Äthiopien waren dagegen religiöse Institutionen zuständig, 
die ihre Maßstäbe anwandten. Panagiotidis zeigt anhand der Akten, wie politisch und bürokratisch um die jeweilige Zugehörigkeit gerungen wurde. Er dementiert die in der Öffentlichkeit und auch in der wissenschaftlichen Literatur verbreiteten Stereotypen einer offenen Tür für alle ethnisch irgendwie Zugehörigen. Es wird deutlich, wie komplex der Prozess des selektiven ,unmixing“ (S. 9) der Bevölkerungen im östlichen Europa und später im Mittleren Osten und in Nordafrika war und wie soziale Zusammenhänge zerschnitten wurden.

Zwei Jahre später legte Panagiotidis ein Überblicksbuch zur ,,postsowjetischen Migration“ nach Deutschland vor. Er füllt damit eine Lücke, denn die größte Einwanderungsgruppe der letzten Jahrzehnte mit 3,5 Millionen Menschen ist in der Öffentlichkeit ebenso wie in der Migrationsliteratur inzwischen ziemlich unsichtbar. Panagiotidis beschreibt ihre Adaptionsprozesse als Erfolg, hauptsächlich wegen der von Anfang an klaren Aufnahmeperspektive und der sofortigen Einbürgerung der Russlanddeutschen. Sprachkurse wurden angeboten und die Aktivität der Einwanderer freigesetzt, obwohl der Arbeitsmarkt in der Haupteinwanderungszeit 1987-2005 wenig aufnahmebereit war und es zu beruflichen Dequalifizierungen kam. Die Haushaltseinkommen sind inzwischen annähernd so hoch wie die der Einheimischen und Menschen wie Helene Fischer und Wladimir Kaminer gehören zum Mainstream.

Panagiotidis arbeitet mit einem kreativen Mix aus systematischer Darstellung, statistischen Analysen und eigenen Feldforschungen, beispielweise einer originellen Analyse der assimilativen Vornamensgebung der Kinder von Russlanddeutschen. Persönliche Erfahrungen und Eindrücke illustrieren die Darstellung. Er zeigt kartografisch, wie Russlanddeutsche Siedlungsschwerpunkte in kleinstädtischen Räumen bilden, hauptsächlich im ostwestfälisch-niedersächsischen Raum und in BadenWürttemberg, im Unterschied zu den jüdischen Kontingentflüchtlingen und anderen Migrationsgruppen, die eher in großen Städten leben. Er beschreibt die familialen und sozialen Lebenswelten, die sich über die Migration hinaus erhalten und gebildet haben, einschließlich der besonderen religiösen Gruppierungen.

Skeptisch beurteilt Panagiotidis die bürokratische Steuerung der Zuwanderung durch das „Wohnortzuweisungsgesetz“, mit dem seit 1989 Konzentrationen verhindert werden sollten. In der Asylkrise wurde diese bürokratische Fixierung als bewährtes Modell betrachtet und auf Flüchtlinge übertragen. Panagiotidis zeigt, dass Aussiedler gleichwohl mehr als andere Einwanderungsgruppen in kleinräumigen lokalen Wohnkonzentrationen leben, ohne dass das ihrer Integration schaden würde.

Die politische Passivität der Russlanddeutschen erklärt Panagiotidis mit dem dominanten Integrationsdiskurs. Sein Hinweis auf die völlig gegensätzliche Situation in Israel mit zwei aktiven russischstämmigen Einwandererparteien dürfte zu der Erklärung führen, dass die Tabuisierung des Nationalismus in Deutschland wenig politischen Raum für die Betonung nationaler Zuwanderung lässt. Russlanddeutsche Spätaussiedler, schreibt Panagiotidis, wurden ,immer wieder zur Projektionsfläche der spannungsgeladenen Auseinandersetzung ,einheimischer“ Deutscher mit der eigenen Konzeption von Deutschsein“ (S. 144). Die Vorurteile verschoben sich dabei in der Haupteinwanderungszeit und Aussiedler waren zeitweise sowohl mit Ausländerfeindlichkeit konfrontiert als auch „Sündenböcke der Linken“ (WolfAlmanasreh). Inzwischen sind die Vorurteile abgeklungen, aber noch auffindbar. Panagiotidis hat das im Dezember 2020 in einem Blog als „Die Schäferhündchen- 
Frage" aufgespießt, als ein bekannter Journalist die denunzierende Formulierung des ehemaligen Berliner Innensenators Lummer von 1988 wiederaufnahm, das einzig Deutsche an manchen Aussiedlern sei der Besitz eines deutschen Schäferhunds.

„Als Migrant existierst Du nur als ,Problem-Migrant““, schreibt Sergej Lagodinsky im Vorwort der ,postsowjetischen Migration“ (S. 10). Eine Gruppe, die sich in Deutschland weitgehend assimiliert und unspektakulär wird, passt nicht in die verbreiteten Klischees migrantischer Hilfsbedürftigkeit, Problematik und Andersartigkeit, die auch in der wissenschaftlichen Literatur verbreitet sind.

Die beiden Bücher werden maßgebend bleiben für die Einwanderungs- und Integrationsgeschichte der ,fremden“ Deutschen und Juden. Sie führen über die Stereotypen hinaus, mit der die ,ethnische Einwanderung“ vielfach nicht nur in der Öffentlichkeit, sondern auch in der Literatur betrachtet worden ist. Wie stark diese beiden Geschichten aufeinander bezogen gewesen sind, macht Panagiotidis deutlich.

Funding Open Access funding enabled and organized by Projekt DEAL.

Open Access Dieser Artikel wird unter der Creative Commons Namensnennung 4.0 International Lizenz veröffentlicht, welche die Nutzung, Vervielfältigung, Bearbeitung, Verbreitung und Wiedergabe in jeglichem Medium und Format erlaubt, sofern Sie den/die ursprünglichen Autor(en) und die Quelle ordnungsgemäß nennen, einen Link zur Creative Commons Lizenz beifügen und angeben, ob Änderungen vorgenommen wurden.

Die in diesem Artikel enthaltenen Bilder und sonstiges Drittmaterial unterliegen ebenfalls der genannten Creative Commons Lizenz, sofern sich aus der Abbildungslegende nichts anderes ergibt. Sofern das betreffende Material nicht unter der genannten Creative Commons Lizenz steht und die betreffende Handlung nicht nach gesetzlichen Vorschriften erlaubt ist, ist für die oben aufgeführten Weiterverwendungen des Materials die Einwilligung des jeweiligen Rechteinhabers einzuholen.

Weitere Details zur Lizenz entnehmen Sie bitte der Lizenzinformation auf http://creativecommons.org/ licenses/by/4.0/deed.de. 